\title{
Blocking painful interactions
}

The neuronal voltage gated calcium channel Cav2.2 is an established contributor to many persistent pain syndromes; however, existing calcium channel blockers are relatively nonspecific and result in undesirable side effects. A paper published in Nature Medicine now reveals a new approach to target the function of Cav2.2 using a small synthetic peptide, alleviating inflammatory and neuropathic pain with minimal adverse effects.

The axonal protein collapsinresponse mediator protein 2 (CRMP2; also known as DRP2) interacts with Cav2.2 and modulates its expression and capacity

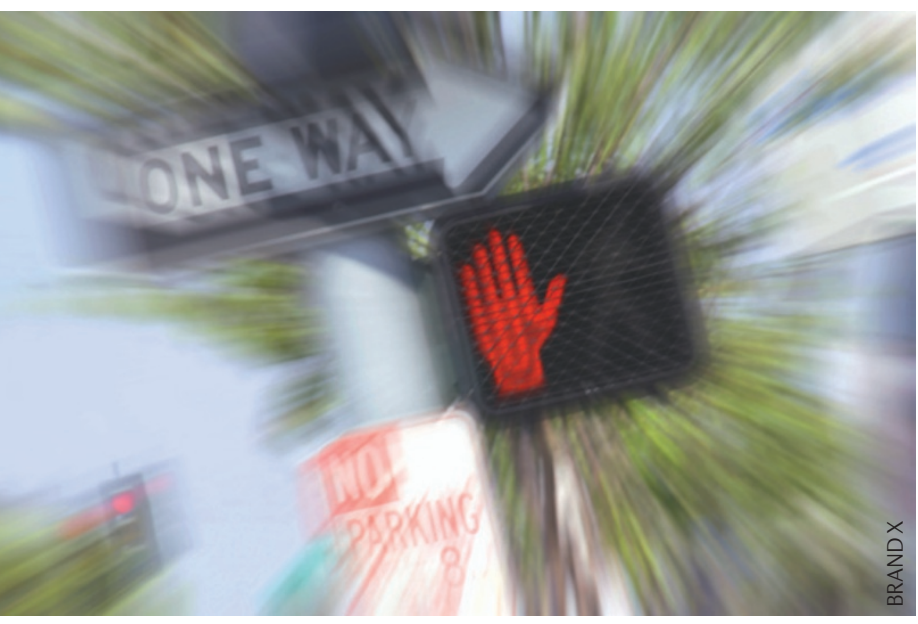

to transmit neuronal excitability. The authors aimed to disrupt this interaction by designing a synthetic peptide (CBD3) that mimics the action of CRMP2 in binding to Cav2.2. When fused to sequences that allowed it to cross the plasma membrane, $\mathrm{CBD} 3$ prevented the interaction of Cav2.2 and CRMP2, decreased the surface expression of Cav2.2 and reduced calcium influx in cultured neurons.

The release of neuropeptides, including calcitonin gene-related peptide (CGRP), from sensory neurons contributes to the transmission of inflammatory pain. The authors found that $\mathrm{CBD} 3$ reduced CGRP release that was evoked by excitation or capsaicin in cultured dorsal root ganglion neurons and spinal cord slices, respectively. Furthermore, $\mathrm{CBD} 3$ reduced the pain-related, CGRP-mediated vasodilation of meningeal blood vessels in rats.

To determine whether these changes in sensory transmission related to a reduction in pain sensitivity, the authors examined the effects of CBD3 in several behavioural assays of acute and chronic pain in rats. CBD3 reduced both formalin- and capsaicin-induced pain behaviours. Furthermore, CBD3 alleviated the tactile hypersensitivity induced by an antiretroviral drug, a model of the chronic pain often experienced by patients being treated for AIDS.

The adverse effects of the currently approved $\mathrm{N}$-type calcium channel blocker ziconotide include memory loss and psychiatric symptoms. It was therefore important to determine whether CBD3 has similar effects. The authors found no evidence of defects in mice treated with the peptide using a battery of neurobehavioural tests. Similarly, the peptide did not induce depressionlike behaviours in mice.

These findings show that a small synthetic peptide that blocks the interaction of CRMP2 with Cav2.2 can attenuate pain transmission and pain-related behaviours in animal models without substantial adverse effects. Future work may take advantage of the new approach outlined here to improve the treatment of intractable clinical pain.

Katherine Whalley

ORIGINAL RESEARCH PAPER Brittain, J. M. et al. Suppression of inflammatory and neuropathic pain by uncoupling CRMP-2 from the presynaptic $\mathrm{Ca}^{2+}$ channel complex. Nature Med. 5 June 2011 (doi:10.1038/nm.2345) 